Applied Mathematical Sciences, Vol. 7, 2013, no. 15, 713 - 716

\title{
Approximation of Stochastic Equilibria for \\ Dynamical Systems with Parametrical Noise
}

\author{
Irina Bashkirtseva \\ Ural Federal University, Ekaterinburg, Russia \\ irina.bashkirtseva@usu.ru
}

\begin{abstract}
A problem of the approximation for stochastic equilibria for the system with parametrical noise is considered. Our approach is based on first approximation stochastic systems technique. For these systems, we use a spectral theory of positive operators for the analysis of exponential mean square stability. Using this method we approximate a dispersion of random states in stochastic equilibrium of nonlinear dynamical system with parametrical noise.
\end{abstract}

Keywords: Stochastic equilibria, parametrical noise, dispersion

\section{Introduction}

A dispersion of random states of stochastic attractor is an important characterisics of the quantitative analysis of the various noise-induced phenomena [1]. For systems forced by additive noise only, the approximations of the dispersions via stochastic sensitivity functions technique were successfully applied to the parametrical analysis of the stochastic phenomena: backward stochastic bifurcations [2], excitability of noisy neuron [3], noise-induced extinction in the population system [4], noise-induced intermittency [5] and chaos [6].

In the present paper, an influence of parametrical noise on the dispersion of random attractors is studied. In Section 1, a necessary mathematical background is given. In Section 2, we apply this theory to the analysis of stochastic equilibria of randomly forced Van der Pol system.

\section{Analysis of stochastic equilibria}

Consider a nonlinear deterministic system

$$
d x=f(x) d t
$$

and a stochastic Ito's system with parametrical noises

$$
d x=f(x) d t+\sum_{i=1}^{k} \sigma_{i}(x) d w_{i} .
$$


Let $\bar{x}$ be an exponentially stable equilibrium of the deterministic system (1). For the deviation $z=x-\bar{x}$ of the random state $x$ of the system (1) from the equilibrium $\bar{x}$, one can write a first approximation system

$$
\begin{aligned}
& d z=F z d t+\sum_{i=1}^{k}\left(S_{1, i}+S_{2, i} z\right) d w_{i} \\
& F=\frac{\partial f}{\partial x}(\bar{x}), \quad S_{1, i}=\sigma_{i}(\bar{x}), \quad S_{2, i}=\frac{\partial \sigma_{i}}{\partial x}(\bar{x}) .
\end{aligned}
$$

The moments $m=\mathrm{E} z, \quad M=\mathrm{E} z z^{\top}$ of the system (3) solutions are governed by equations:

$$
\begin{gathered}
\dot{m}=F m, \\
\dot{M}=F M+M F^{\top}+\sum_{i=1}^{k}\left(S_{1, i} S_{1, i}^{\top}+S_{1, i} m^{\top} S_{2, i}^{\top}+S_{2, i} m S_{1, i}^{\top}+S_{2, i} M S_{2, i}^{\top}\right) .
\end{gathered}
$$

Our interest is focused on the stationary solutions of (4), (5). A problem of the existence of these stationary solutions is connected with the theory of the mean square stability $[7,8]$.

Consider a homogenious linear system

$$
d z=F z d t+\sum_{i=1}^{m} S_{2, i} z d w_{i}
$$

and operators

$$
\mathcal{A}(M)=F M+M F^{\top}, \quad \mathcal{S}(M)=\sum_{i=1}^{m} S_{2, i} M S_{2, i}^{\top}, \quad \mathcal{P}=-\mathcal{A}^{-1} \mathcal{S} .
$$

Theorem 1. The system (4),(5) has an exponentially stable stationary solution $(\bar{m}, \bar{M})$ if and only if a solution $z \equiv 0$ of the system (6) is exponentially mean square stable. In this case, $\bar{m}=0$, and $\bar{M}$ is a unique solution of the matrix algebraic equation

$$
F M+M F^{\top}+S_{1}+\mathcal{S}(M)=0, \quad S_{1}=\sum_{i=1}^{k} S_{1, i} S_{1, i}^{\top} .
$$

Here the following algebraic criterion holds:

Theorem 2. The solution $z \equiv 0$ of the system (6) is exponentially mean square stable iff $\operatorname{Re} \lambda_{i}(F)<0$ and $\rho(\mathcal{P})<1$, where $\lambda_{i}(F)$ are eigenvalues of the matrix $F$, and $\rho(\mathcal{P})$ is a spectral radius of the operator $\mathcal{P}$.

Main ideas of the proof of the Theorem 2 can be found in $[9,10]$. The Theorem 2 implies the Theorem 1.

The matrix $M$ can be used as an approximation of covariance matrix for the stationary distributed random states of the stochastic equilibria of the nonlinear system (2). 
2. Example. Consider stochastically forced Van der Pol oscillator

$$
\begin{aligned}
& \dot{x}=y \\
& \dot{y}=-x+a\left(1-x^{2}\right) y+\sigma_{1} \dot{w}_{1}+\sigma_{2} y \dot{w}_{2} .
\end{aligned}
$$

Here, $\sigma_{1}$ is the intensity of additive noise and $\sigma_{2}$ is the intensity of parametrical noise, $w_{1,2}$ are independent Wiener processes.

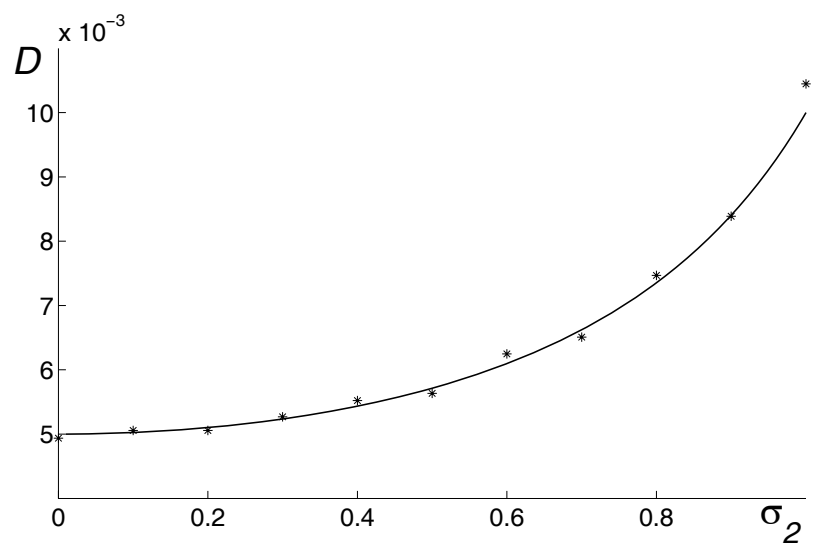

Fig. 1. Dispersion of $x$-coordinate of the stochastic equilibrium of the Van der Pol oscillator for $a=-1, \sigma_{1}=0.1$.

The deterministic system $\left(\sigma_{1}=\sigma_{2}=0\right)$ exhibits a stable equilibrium $\bar{x}=0, \bar{y}=0$. Under stochastic disturbances, random trajectories leave the stable equilibrium and form a stationary probabilistic distribution (stochastic equilibrium). We are interested in the dispersion $D$ of $x$-coordinate of this stochastic equilibrium.

As noise intensity increases, the function $D$ increases too. Results of the direct numerical simulation of the function $D\left(\sigma_{2}\right)$ for the system (8) with $a=-1, \sigma_{1}=0.1$ are plotted in Fig. 1 by asterisks.

For the analytical approximation of the dispersion, we will use the first approximation stochastic systems technique presented above.

Parameters of the equation (7) for the system (8) are following:

$$
F=\left[\begin{array}{cc}
0 & 1 \\
-1 & a
\end{array}\right], S_{1}=\sigma_{1}^{2}\left[\begin{array}{ll}
0 & 0 \\
0 & 1
\end{array}\right], M=\left[\begin{array}{ll}
m_{11} & m_{12} \\
m_{21} & m_{22}
\end{array}\right], \mathcal{S}(M)=\sigma_{2}^{2}\left[\begin{array}{cc}
0 & 0 \\
0 & m_{22}
\end{array}\right] .
$$

The equation (7) for the elements $m_{i j}$ of the matrix $M$ can be written as a system:

$m_{12}=m_{21}=0, \quad-m_{11}+a m_{12}+m_{22}=0, \quad-2 m_{12}+2 a m_{22}+\sigma_{1}^{2}+\sigma_{2}^{2} m_{22}=0$.

It follows from this system that

$$
m_{11}=m_{22}=-\frac{\sigma_{1}^{2}}{2 a+\sigma_{2}^{2}}, \quad m_{12}=m_{21}=0 .
$$


This matrix is diagonal and $m_{11}=m_{22}$. We will use the function $m_{11}$ for the approximation of the dispersion $D$ of $x$-coordinate of stochastic equilibrium for the randomly forced nonlinear Van der Pol system. In Fig.1, the function $m_{11}\left(\sigma_{2}\right)$ is plotted by solid line. As one can see, our approximation agrees with the results of direct numerical simulation.

Acknowledgements. The study was supported by the Ministry of education and science of Russia, projects 1.1099.2011, 14.A18.21.0364.

\section{References}

1. W. Horsthemke, R. Lefever, Noise-Induced Transitions, Springer, Berlin (1984).

2. I. Bashkirtseva, L. Ryashko, P. Stikhin, Noise-induced backward bifurcations of stochastic 3D-cycles, Fluctuation and Noise Letters, 9 (2010), 89-106.

3. L. Ryashko, I. Bashkirtseva, Analysis of excitability for the FitzHughNagumo model via a stochastic sensitivity function technique, Phys. Rev. E, 83 (2011), 061109.

4. L. Ryashko, I. Bashkirtseva, Stochastic sensitivity analysis of noiseinduced excitement in a prey-predator plankton system, Frontiers in Life Science, 5 (2011), 141-148.

5. I. Bashkirtseva, L. Ryashko, Stochastic sensitivity analysis of noiseinduced intermittency and transition to chaos in one- dimensional discrete-time systems, Physica A, 392 (2013), 295-306.

6. I. Bashkirtseva, G. Chen and L. Ryashko, Analysis of noise-induced transitions from regular to chaotic oscillations in the Chen system, Chaos, 22 (2012), 033104.

7. R. Z. Khasminskii, Stochastic stability of differential equations, Sijthoff \& Noordhoff, Alpen aan den Rijn (1980).

8. X. Mao, Exponential stability of stochastic differential equations, Marcel Dekker (1994).

9. L. B. Ryashko, Stabilization of linear systems with multiplicative perturbations and incomplete information, J. Appl. Math. Mech., 45 (1981), 581-587.

10. L. Ryashko, I. Bashkirtseva, Exponential Mean Square Stability Analysis of Invariant Manifolds for Nonlinear SDE's, Chapter 4 in: Stochastic Differential Equations, Ed. N. Halidias, Series: Mathematics Research Developments, pp.67-95, Nova Science Publishers (2011).

\section{Received: November, 2012}

